\title{
Metric in Feature Space
}

\author{
C.A. Murthy and Sourav Pradhan \\ Machine Intelligence Unit, Indian Statistical Institute, Kolkata- 700108, India \\ \{murthy, sourav_r\}@isical.ac.in
}

\begin{abstract}
In this paper our purpose is to define a metric in feature space. The metric finds the correlation distance between two features. It may be used in many applications. An application showing the utility of the proposed metric for feature selection is described here. The performance of the feature selection method is found to be comparable to other feature selection methods.
\end{abstract}

Keywords: metric, correlation, PCA, partition, entropy, regression.

\section{Introduction}

In pattern recognition literature, to evaluate the significance of features, many measures and their variations like distance measures [1], consistency measures [2], dependency measure [3], mutual information measures [4], classification error [5] have been introduced. But there are very few measures to find distance between two features. Mitra et al. [6] defined an index called maximal information compression index, which is like a distance measure between two features. This measure is invariant to translation but sensitive to scaling. In this present article we will define a distance measure which is invariant to both translation and scaling. This distance measure may be used effectively in several contexts. Here we have used it in feature selection.

\section{Definitions and Basic Mathematics}

This section provides the definitions and basic mathematics regarding features and metric. We shall assume that $\Omega$ is the space under consideration.

Definition 1 (Feature). A feature $X$ is a function from $\Omega$ to $\mathbb{R}$.

$$
X: \Omega \rightarrow \mathbb{R} .
$$

Let $S$ be the set of all features.

Definition 2 (Metric). For a nonempty set $M, \rho$ is said to be a metric on $M$ if $\rho: M \times M \longrightarrow \mathbb{R}$ such that

(i) $\rho(x, y) \geq 0$

$\forall x, y \in M$ (non-negativity)

(ii) $\rho(x, y)=0$

if and only if $x=y$

S. Chaudhury et al. (Eds.): PReMI 2009, LNCS 5909, pp. 50-55 2009.

(C) Springer-Verlag Berlin Heidelberg 2009 
(iii) $\rho(x, y)=\rho(y, x) \quad \forall x, y \in M$ (symmetry)

(iv) $\rho(x, z) \leq \rho(x, y)+\rho(y, z) \quad \forall x, y, z \in M \quad$ (triangular inequality)

If $\Omega$ is a finite set then we can have

$$
l_{m}(X, Y)=\left(\sum_{w \in \Omega}|X(w)-Y(w)|^{m}\right)^{1 / m} \quad \forall m \geq 1 ; \quad X, Y \in S
$$

If $\Omega$ is an uncountable set and integration is possible then we can have

$$
L_{m}(X, Y)=\left(\int_{\Omega}|X(w)-Y(w)|^{m} d w\right)^{1 / m} \quad \forall m \geq 1
$$

Note that $l_{m}$ and $L_{m}$ are metrics for every $m \geq 1$ and they are known as Minkowski metrics.

One can compute the distance between two features using these metrics but results may or may not be meaningful. For example, if the feature space is transformed by multiplying a feature vector by an arbitrary constant, then distance relationships in the transformed space can be very different from the original distance relationships, even though the transformation merely amounts to a different choice of units for the feature. Such scale changes can have a major impact on feature subset selection problem. We would like to make the distance function between two features to be translation and scale invariant. So we want that the following two properties be satisfied by the feature distance measure denoted by $d$.

P1. $d(X, a X+b)=0 \quad \forall a \in \mathbb{R}, a \neq 0$ and $\forall b \in \mathbb{R}$

P2. $d(X, Y)=d(a X+b, Y) \quad \forall a \in \mathbb{R}, a \neq 0$ and $\forall b \in \mathbb{R}$

where $X$ and $Y$ are two features. First property says that features $X$ and $a X+b$ are equivalent. Because feature $X$ is scaled and translated to $a X+b$. As features $X$ and $a X+b$ are equivalent then distances from $Y$ to $X$ and $a X+b$ must be same. For proper mathematical formulation of the above two properties let $S$ be the set of all features. Let

$$
S_{X}=\{a X+b: \quad a \in \mathbb{R}, a \neq 0 \text { and }, b \in \mathbb{R}\}
$$

Proposition. For any two features $X$ and $Y$ either

1. $S_{X} \cap S_{Y}=\emptyset$ or

2. $S_{X}=S_{Y}$

$S_{X}$ 's form a partition of $S$.

The proposition can easily be proved. The following property is also needed to be satisfied

P3. $d(X, Y)=d\left(X_{1}, Y_{1}\right)$ if $X_{1} \in S_{X}$ and $Y_{1} \in S_{Y}$

We would like to make the distance measure to be invariant to scaling and translation of the variables. 
One property of any metric $\rho$ is

$$
\rho(x, y)=0 \text { if and only if } x=y \text {; }
$$

Note that $d$ does not satisfy it since

$$
d(X, a X+b)=0 \quad \forall a \in \mathbb{R}, a \neq 0 \text { and }, \forall b \in \mathbb{R}
$$

Thus to make proper mathematical formulation, let us assume that $\mathcal{A}=\left\{S_{X}\right.$ : $X \in S\}$. Note that $\mathcal{A}$ is the set of all partitions created by $S_{X}$. Let distance function $D$ be defined on $\mathcal{A}$, i.e., $D: \mathcal{A} \times \mathcal{A} \longrightarrow[0, \infty)$. Let

$$
D\left(S_{X}, S_{Y}\right)=d(X, Y)
$$

where $d$ satisfies properties $\boldsymbol{P} \mathbf{1}, \boldsymbol{P} \mathscr{2}$, and $\boldsymbol{P}$ 3 stated above. Then metric property (ii) is satisfied if $d$ is defined properly. One of the quantities which is translation and scale invariant is $\left|r_{X Y}\right|$ where

$$
r_{X Y}=\frac{\operatorname{cov}(\mathrm{X}, \mathrm{Y})}{\sqrt{\operatorname{var}(\mathrm{X}) \operatorname{var}(\mathrm{Y})}} . \quad-1 \leq r \leq 1
$$

$\operatorname{var}()$ denotes the variance of a variable and $\operatorname{cov}()$ the covariance between two variables. A possible way of defining metric is to make it a function of $r$. In fact $d(X, Y)=\sqrt{1-r_{X Y}}$ can be shown to satisfy triangular inequality. But $d(X, a X+b) \neq 0 \quad \forall a \in \mathbb{R}$ and, $a \neq 0$. Thus we have not considered the following distance measures.

(i) $d(X, Y)=\sqrt{1-r_{X Y}}$

(ii) $d(X, Y)=1-r_{X Y}$

Some of the functions which don't depend on the sign of $r$ are

(i) $d(X, Y)=\sqrt{1-r_{X Y}^{2}}$

(ii) $d(X, Y)=1-\left|r_{X Y}\right|$

In this article we shall concentrate on these two distance measures. The above functions follow the properties $\boldsymbol{P} \mathbf{1}, \boldsymbol{P} \mathscr{2}$, and $\boldsymbol{P}$ 3. The functions also follow (i), (ii), (iii) properties of metric. But triangular inequality has not yet been proved. We have tested for triangular inequality property on 10 lakh data sets, each data set having 10 ordered triples of points, generated randomly from $(0,1)$. The triangular inequality is experimentally verified in each of these 10 lakh data sets for each of the above two d's. For both functions, namely, $d(X, Y)=\sqrt{1-r_{X Y}^{2}}$ and $d(X, Y)=1-\left|r_{X Y}\right|$, the triangular inequality is experimentally seen to be true.

We have tested the utility of the proposed distance measures in feature selection problem. The algorithm for feature selection and the experimental results are given in next section. 


\section{Feature Selection Algorithm, Results and Comparison}

Algorithm. Let the original number of features be $N$, and the original feature set be $O=\left\{F_{i}, i=1, \cdots, N\right\}$. Represent the dissimilarity between the features $F_{i}$ and $F_{j}$ by the distance function $d\left(F_{i}, F_{j}\right)$. Higher the value of $d$ is, the more dissimilar are the features. Let $t_{i}^{k}$ represents the dissimilarity between feature $F_{i}$ and its kth nearest neighbor feature in $R$. Then

Step 1: Choose an initial value of $k \leq(N-1)$. Set the number of iteration iter $\leq\left\lfloor\frac{N}{k}\right\rfloor$. Initialize the reduced feature subset $R$ to the original feature set $O$, i.e., $R \longleftarrow O$.

Step 2: Compute $d\left(F_{i}, F_{j}\right) \quad \forall i, j=1, \ldots, N$

Step 3: For each feature $F_{i} \in R$, compute $t_{i}^{k}$.

Step 4: Find the feature $F_{i^{\prime}}$ for which $t_{i^{\prime}}^{k}$ is minimum. Retain this feature in $R$ and discard $k$ nearest features of $F_{i^{\prime}}$.

(Note: $F_{i^{\prime}}$ denotes the feature for which removing $k$ nearest neighbors will cause minimum error among all the features in $R$ ).

Step 5: If iter $>0$

iter $=$ iter -1 ;

Go to Step 3.

else

Return feature set $R$ as the reduced feature set.

Stop.

Results and Comparison. For our experiment we have used 6 real-life public domain data sets [7] from three different categories: low-dimensional $(D \leq 10)$ (Iris, Wisconsin Breast Cancer), medium-dimensional $(10<D \leq 100)$ (Ionosphere, Spambase), and high-dimensional $(D>100)$ (Multiple features, Isolet), containing both large and relatively smaller number of points.

Four indices, namely, entropy (E) [1], fuzzy feature evaluation index (FFEI) [6], class separability (S) [5], and K-Nearest Neighbor (K-NN) accuracy [6] are considered to demonstrate the efficiency of the proposed methodology and for comparing it with other methods. Representation entropy (RE) [5] is used to show the redundancy present in reduced feature subset. Five feature selection schemes considered for comparison are branch and bound (BB) 5, sequential forward search(SFS) [5], sequential floating forward search (SFFS) [1], and stepwise clustering (SWC)(using correlation coefficient) [6], relief-F [1]. We have got exactly same result for each data set for the two proposed distance functions. The performance of proposed method is also compared with other two feature similarities least square regression error [6] and, maximal information compression index [6] using our algorithm. In our experiment, we have used interclass distance [5] measure as the feature selection criterion for BB, SFS, SFFS algorithms. Following table provide such a comparative result corresponding to high, medium, and low-dimensional data sets when the size of the reduced feature subset is taken to be about half of the original size. Since the branch and bound (BB) and sequential floating forward search (SFFS) algorithms require infeasible 
Table 1. Comparison of Feature Selection Algorithms for real-life data sets

\begin{tabular}{|c|c|c|c|c|c|c|c|}
\hline Data Set & Method & $\mathrm{RE}$ & $\mathrm{E}$ & $\overline{\text { FFEI }}$ & $\mathrm{S}$ & $\begin{array}{l}\text { KNN Ac } \\
\text { Mean (\%) }\end{array}$ & $\begin{array}{l}\text { uuracy } \\
\text { SD (\%) }\end{array}$ \\
\hline \multirow[b]{2}{*}{ Isolet } & $\sqrt{1-r_{X Y}^{2}}$ & 5.99 & 0.87 & 0.42 & 0.07 & 77.73 & 1.26 \\
\hline & $\begin{array}{c}\text { Least Square } \\
\text { Regression error }\end{array}$ & 5.40 & 0.88 & 0.42 & 0.05 & 75.75 & 1.17 \\
\hline \multirow{4}{*}{$\begin{array}{c}\mathrm{N}=617 \\
\mathrm{~d}=309 \\
\mathrm{c}=26 \\
\mathrm{k}=7\end{array}$} & $\begin{array}{c}\text { Maximal Information } \\
\text { Compression Index }\end{array}$ & 5.12 & 0.88 & 0.42 & 0.05 & 70.55 & 1.29 \\
\hline & SWC & 5.98 & 0.87 & 0.42 & 0.06 & 78.25 & 1.22 \\
\hline & Relief-F & 4.68 & 0.90 & 0.43 & 0.03 & 80.32 & 0.93 \\
\hline & SFS & 4.73 & 0.92 & 0.44 & 0.03 & 74.45 & 1.20 \\
\hline \multirow{6}{*}{$\begin{array}{l}\text { Multiple } \\
\text { Features }\end{array}$} & $\sqrt{1-r_{X Y}^{2}}$ & 3.83 & 0.01 & 0.38 & 0.10 & 87.87 & 0.01 \\
\hline & $\begin{array}{c}\text { Least Square } \\
\text { Regression Error }\end{array}$ & 3.75 & 0.02 & 0.38 & 0.10 & 87.12 & 0.01 \\
\hline & $\begin{array}{c}\text { Maximal Information } \\
\text { Compression Index }\end{array}$ & 3.2 & 0.02 & 0.37 & 0.08 & 81.54 & 0.02 \\
\hline & SWC & 0.24 & 0.02 & 0.33 & 0.02 & 72.62 & 0.01 \\
\hline & Relief-F & 3.36 & 0.02 & 0.38 & 0.08 & 86.35 & 0.01 \\
\hline & SFS & 0.61 & 0.02 & 0.33 & 0.02 & 79.35 & 0.02 \\
\hline \multirow{8}{*}{ Spambase } & $\sqrt{1-r_{X Y}^{2}}$ & 0.01 & 0.01 & 0.03 & 9.34 & 75.68 & 0.79 \\
\hline & $\begin{array}{c}\text { Least Square } \\
\text { Regression error }\end{array}$ & 0.39 & 0.01 & 0.04 & 7.10 & 70.64 & 0.92 \\
\hline & $\begin{array}{c}\text { Maximal Information } \\
\text { Compression Index }\end{array}$ & 0.03 & 0.01 & 0.04 & 6.92 & 68.60 & 0.59 \\
\hline & SWC & 0.01 & 0.01 & 0.03 & 9.34 & 76.40 & 1.05 \\
\hline & Relief-F & 0.15 & 0.01 & 0.03 & 9.53 & 79.08 & 0.90 \\
\hline & SFS & 0.39 & 0.01 & 0.04 & 7.10 & 70.73 & 0.77 \\
\hline & SFFS & 0.39 & 0.01 & 0.04 & 7.10 & 70.73 & 0.77 \\
\hline & $\mathrm{BB}$ & 0.38 & 0.01 & 0.03 & 7.11 & 70.93 & 0.70 \\
\hline \multirow{8}{*}{ Ionosphere } & $\sqrt{1-r_{X Y}^{2}}$ & 3.36 & 0.78 & 0.41 & 5.65 & 73.75 & 5.46 \\
\hline & $\begin{array}{c}\text { Least Square } \\
\text { Regression Error }\end{array}$ & 3.21 & 0.80 & 0.42 & 30.73 & 70.60 & 4.58 \\
\hline & $\begin{array}{c}\text { Maximal Information } \\
\text { compression Index }\end{array}$ & 3.31 & 0.80 & 0.41 & $\overline{12.16}$ & 70.60 & 4.58 \\
\hline & SWC & 3.38 & 0.78 & 0.41 & 11.59 & 71.39 & 5.27 \\
\hline & Relief-F & 3.18 & 0.81 & 0.42 & 8.21 & 71.58 & 6.36 \\
\hline & SFS & 2.68 & 0.82 & 0.42 & 11.35 & 70.73 & 7.23 \\
\hline & SFFS & 2.68 & 0.82 & 0.42 & 11.35 & 70.73 & 7.23 \\
\hline & $\overline{\mathrm{BB}}$ & 3.18 & 0.81 & 0.42 & 11.20 & 72.14 & 7.50 \\
\hline \multirow{9}{*}{ Cancer } & $\sqrt{1-r_{X Y}^{2}}$ & 1.46 & 0.37 & 0.35 & 0.31 & 94.32 & 1.90 \\
\hline & Least Square & 1.51 & 0.37 & 0.36 & 0.34 & 94.25 & 0.89 \\
\hline & Regression error & & & & & & \\
\hline & $\begin{array}{c}\text { Maximal Information } \\
\text { Compression Index }\end{array}$ & 1.53 & 0.38 & 0.35 & 0.33 & 94.19 & 1.50 \\
\hline & SWC & 1.46 & 0.37 & 0.35 & 0.31 & 95.03 & 0.94 \\
\hline & Relief-F & 1.44 & 0.39 & 0.35 & 0.29 & 95.57 & 0.54 \\
\hline & SFS & 1.23 & 0.36 & 0.33 & 0.26 & 95.65 & 0.47 \\
\hline & SFFS & 1.23 & 0.36 & 0.33 & 0.26 & 95.65 & 0.47 \\
\hline & $\mathrm{BB}$ & 1.23 & 0.36 & 0.33 & 0.26 & 95.65 & 0.47 \\
\hline \multirow{9}{*}{ Iris } & $\sqrt{1-r_{X Y}^{2}}$ & 0.27 & 0.35 & 0.56 & 0.03 & 93.48 & 2.03 \\
\hline & Least Square & 0.23 & 0.57 & 0.35 & 0.04 & 92.29 & 2.57 \\
\hline & Regression Error & & & & & & \\
\hline & $\begin{array}{c}\text { Maximal Information } \\
\text { Compression Index }\end{array}$ & 0.73 & 0.58 & 0.37 & 0.09 & 93.03 & 2.50 \\
\hline & SWC & 0.27 & 0.56 & 0.35 & 0.03 & 93.48 & 2.03 \\
\hline & Relief-F & 0.08 & 0.58 & 0.34 & 0.02 & 94.44 & 3.71 \\
\hline & SFS & 0.23 & 0.57 & 0.35 & 0.05 & 92.29 & 2.57 \\
\hline & SFFS & 0.23 & 0.57 & 0.35 & 0.05 & 92.29 & 2.57 \\
\hline & $\mathrm{BB}$ & 0.23 & 0.57 & 0.35 & 0.05 & 92.29 & 2.57 \\
\hline
\end{tabular}


high computation time for the large data sets, we could not provide the results for them in the table. For the classification accuracy using K-NN, both the mean and standard deviations (SD) computed for ten independent runs are presented. In relating to the search-based algorithms (BB, SFFS, and SFS) and two feature similarities (least square regression error and maximal information compression index), the performance of the proposed method is comparable or sometimes better. In a part of the experiment, we compared the performance with a supervised method Relief-F, which is widely used. We have used 50 percent of the samples as design set for the Relief-F algorithm. Sometimes, the Relief-F algorithm provides classification performance better than our method but its performance in terms of the unsupervised indices is poor. Relief-F has a higher time requirement, specially for data sets with large number of samples because the number of iterations is huge. The performance of our method is better, in terms of unsupervised indices, than least square regression error and maximal information compression index. Representation entropy performance for proposed method is comparable to other methods but better for large dimensional data sets.

\section{Conclusion}

A distance measure, invariant to translation and scaling, is proposed for feature subset selection. This measure is fast to compute as computational complexity for finding correlation between two variables is linear. The performance of this distance measure using the algorithm described in this paper is comparable to other methods, generally better for unsupervised case.

\section{Acknowledgement}

The authors wish to thank Mr. B. L. Narayan, Yahoo India, Bangalore for his helping in this work.

\section{References}

1. Liu, H., Motoda, H.: Computational Methods of Feature Selection. Chapman and Hall/Crc Data Mining and Knowledge Discovery Series (2007)

2. Dash, M., Liu, H.: Consistency-based search in feature selection. Artif. Intell. 151(12), 155-176 (2003)

3. Liu, H., Yu, L.: Toward integrating feature selection algorithms for classification and clustering. IEEE Trans. Knowl. Data Eng. 17(4), 491-502 (2005)

4. Estévez, P.A., Tesmer, M., Perez, C.A., Zurada, J.M.: Normalized mutual information feature selection. IEEE Trans. on Neural Networks 20(2), 189-201 (2009)

5. Devijver, P., Kittler, J.: Pattern Recognition: A Statistical Approach. Prentice Hall, Engle Cliffs (1982)

6. Mitra, P., Murthy, C.A., Pal, S.K.: Unsupervised Feature Selection Using Feature Similarity. IEEE Trans. Pattern Analysis and Machine Intelligence 24(3), 301-312 (2002)

7. Blake, C.L., Merz, C.J.: UCI Repository of Machine Learning Database, Univ. of California, Irvine, Dept. of Information and Computer Science (1998) 